\title{
Thermal Strain Measured by Fiber Bragg Grating Sensors
}

\author{
Shiuh-Chuan Her* and Chih-Ying Huang \\ Department of Mechanical Engineering, Yuan Ze University, 135 Yuan-Tung Road, Chung-Li 320, Taiwan
}

(Received December 22, 2015; accepted May 20, 2016)

Keywords: thermal strain, fiber Bragg grating, optic-fiber sensor

An analytical solution is presented to predict the thermal stress and strain induced by a temperature change on an optic-fiber sensor surface bonded onto a host structure. It shows that the thermal strain transferred from the host material to the optic-fiber is heavily affected by the bonding characteristics including the optic-fiber, protective coating, adhesive and host material. Experimental tests were conducted using a fiber Bragg grating sensor to evaluate the thermal strain on the surface-bonded optic fiber. The accuracy of the theoretical prediction was validated by the experimental results. Experimental measurements matched well with the analytical predictions.

\section{Introduction}

Optical fiber sensors have the advantages of light weight, small size, high resolution, accuracy, and immunity to electromagnetic interference. These sensors have been employed in a variety of engineering applications such as health monitoring of composite structures, ${ }^{(1)}$ operational load monitoring of wind turbine blades, ${ }^{(2)}$ nondestructive evaluation, and damaging detection. ${ }^{(3)}$ They are helpful because they enable direct measurement of strain in the interior of a host structure at critical locations, thus providing useful information about the high strain state in a complicated structure. Optic-fiber sensors can be classified into three different types of sensors based on the light parameters which are modulated, namely, wavelength, phase, and intensity. Fiber Bragg grating (FBG) sensors based on wavelength modulation have been widely used in the monitoring of structural health. Sorensen et al. ${ }^{(4)}$ employed FBG sensors to evaluate the residual strain in AS4/ PPS thermoplastic composites. Schizas and Karalekas ${ }^{(5)}$ used FBG sensors to measure solidification strain in a photocurable resin. Yashiro and Okabe ${ }^{(6)}$ estimated fatigue damage in composite laminates with a hole using an embedded FBG sensor. To investigate structural shape and deformation, Kang et al.(7) employed FBG sensors to measure dynamic structural displacements. Torres et al ${ }^{(8)}$ presented a new FBG strain sensor with an unsymmetrical packaging configuration to be fixed to the surface of a structure for structural health monitoring. Li et al..$^{(9)}$ developed a novel FBG sensor for use in monitoring the force on bridge cables. They employed this new type of FBG force sensor to detect the cable forces in an arch bridge structure. Their study showed that the new FBG force sensor exhibited better accuracy in cable force measurements and that temperature effects could be appropriately compensated. Shrestha et al. ${ }^{(10)}$ proposed a 1-dimensional (1D) FBG sensor array configuration for impact localization of random impact points on a composite wing. Their study showed that two FBG sensors covering a wing area of $4.2 \times 0.6 \mathrm{~m}^{2}$ could approximate the location of impact with a maximum localization error limited to about $35.0 \mathrm{~mm}$. For concrete "Corresponding author: e-mail: mesch@saturn.yzu.edu.tw 
structures, corrosion is a critical problem that significantly reduces the service life of civil

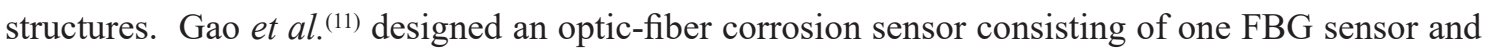
twin steel rebar elements packaged with concrete to detect rebar corrosion. Jiang et al. ${ }^{(12)}$ presented a new optic-fiber sensor for the simultaneous measurements of liquid level and surrounding refractive index based on a tilted FBG. It is well known that the interferometric types of opticfiber sensors have the advantages of high sensitivity and spatial resolution. Thus, they have been extensively used in composite structures for health monitoring. ${ }^{(1,13)}$

In this study, the optic-fiber sensor was bonded to the surface of a host structure. A closeform expression of the thermal stress and strain in the surface bonded optic-fiber resulting from a temperature change was presented. Theoretical predictions were verified through a series of experimental tests involving FBG sensors.

\section{Thermal Analysis}

The geometrical model for the thermal analysis is presented in Fig. 1. It consisted of four different materials: the optic-fiber, protective coating, adhesive layer, and host structure. In this study, thermal analysis was carried out on the four-material composite structure for a uniform temperature change $\Delta T$. Thermal stresses developed in the composite structure as a result of differences in the thermal expansion coefficients between the optic fiber and host material. Part of the thermal strain was transferred from the host material to the optic fiber through the adhesive layer and protective coating, while the composite structure was subjected to a uniform temperature change. The thermal stress and strain of the surface-bonded optic fiber derived by Her and Huang ${ }^{(14)}$ based on the shear lag model are defined as follows:

$$
\sigma_{1}^{\mathrm{f}}=\frac{\left(\alpha_{\mathrm{h}}-\alpha_{\mathrm{f}}\right) \Delta T}{\left(\frac{\pi r_{\mathrm{f}}^{2}}{2 h r_{\mathrm{p}} E_{\mathrm{h}}}+\frac{1}{E_{\mathrm{f}}}\right)}\left[1-\frac{\cosh \left(\lambda_{1} x\right)}{\cosh \left(\lambda_{1} L_{\mathrm{f}}\right)}\right]
$$

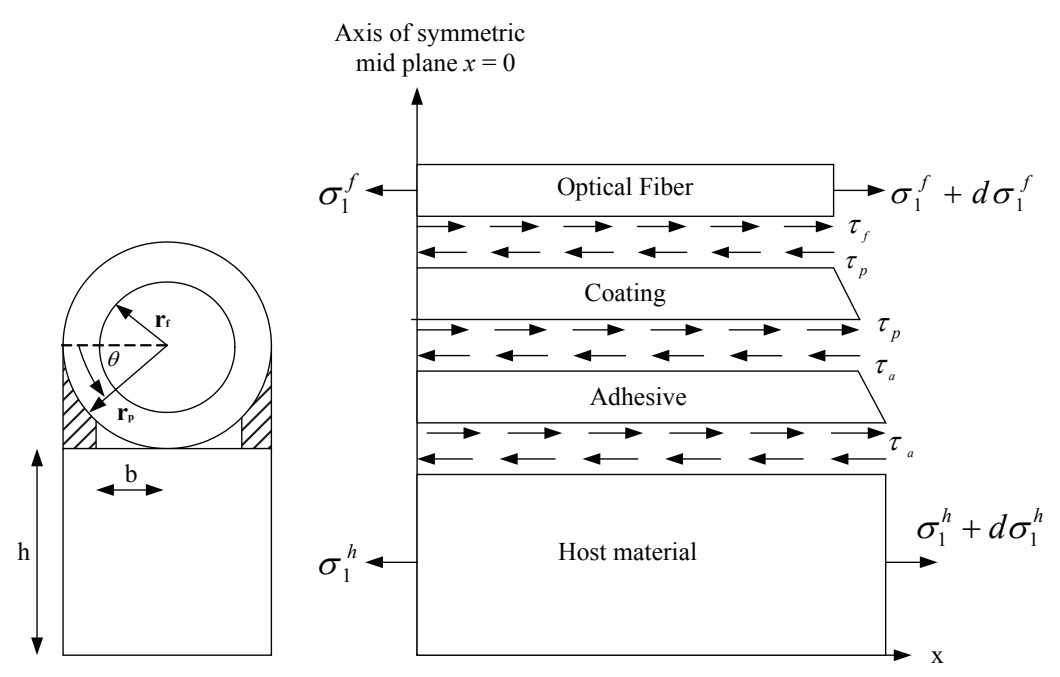

Fig. 1. Geometrical model of surface-bonded optic fiber. ${ }^{(14)}$ 


$$
\begin{gathered}
\varepsilon_{1}^{\mathrm{f}}=\frac{\left(\alpha_{\mathrm{h}}-\alpha_{\mathrm{f}}\right) \Delta T}{E_{\mathrm{f}}\left(\frac{\pi r_{\mathrm{f}}^{2}}{2 h r_{\mathrm{p}} E_{\mathrm{h}}}+\frac{1}{E_{\mathrm{f}}}\right)}\left[1-\frac{\cosh \left(\lambda_{1} x\right)}{\cosh \left(\lambda_{1} L_{\mathrm{f}}\right)}\right]+\alpha_{\mathrm{f}} \Delta T \\
\lambda_{1}=\sqrt{\frac{2 r_{\mathrm{p}}}{\pi r_{\mathrm{f}}^{2}}\left(\frac{\pi r_{\mathrm{f}}^{2}}{2 h r_{\mathrm{p}} E_{\mathrm{h}}}+\frac{1}{E_{\mathrm{f}}}\right) \int_{0}^{\cos ^{-1}\left(\frac{b_{\mathrm{p}}}{r_{\mathrm{p}}}\right)} \frac{1}{\frac{r_{\mathrm{p}}(1-\sin \theta)}{G_{\mathrm{a}}}+\frac{r_{\mathrm{p}}}{G_{\mathrm{p}}} \ln \left(\frac{r_{\mathrm{p}}}{r_{\mathrm{f}}}\right)} d \theta}
\end{gathered}
$$

The average of the thermal strain in the optic fiber along the bonding length due to the temperature change is

$$
\left(\varepsilon_{1}^{\mathrm{f}}\right)_{\text {avg }}=\frac{\int_{\Gamma_{\mathrm{s}}} \varepsilon_{1}^{\mathrm{f}}(x) d x}{L_{\mathrm{f}}}=\frac{\left(\alpha_{\mathrm{h}}-\alpha_{\mathrm{f}}\right) \Delta T}{\mathrm{E}_{\mathrm{f}}\left(\frac{\pi r_{\mathrm{f}}^{2}}{2 h r_{\mathrm{p}} E_{\mathrm{h}}}+\frac{1}{E_{\mathrm{f}}}\right)}\left[1-\frac{\sinh \left(\lambda_{1} L_{\mathrm{f}}\right)}{\lambda_{1} L_{\mathrm{f}} \cosh \left(\lambda_{1} L_{\mathrm{f}}\right)}\right]+\alpha_{\mathrm{f}} \Delta T,
$$

where $r_{\mathrm{p}}$ and $r_{\mathrm{f}}$ denote the radii of the coating and optic-fiber, respectively; $\alpha_{\mathrm{p}}$ and $\alpha_{\mathrm{f}}$ represent the coefficients of the thermal expansion of the protective coating and optic-fiber, respectively; $E_{\mathrm{f}}$ and $E_{\mathrm{h}}$ are the Young's moduli of the optic-fiber and host structure, respectively; $G_{\mathrm{p}}$ and $G_{\mathrm{a}}$ represent the shear moduli of the coating and adhesive, respectively; and $L_{\mathrm{f}}$ and $h$ are the half of the bonding length and thickness of the host material, respectively.

\section{FBG Sensor}

FBG sensors are formed by modulating the index of refraction along a length of the core of an optic fiber. The modulation is conducted by exposing the specific region of the optical fiber (FBG region) to UV radiation. When the light of a broadband spectrum is passed through the optical fiber to the FBG, a narrow band is reflected back at the Bragg resonance wavelength $\lambda_{\mathrm{B}}$, given by

$$
\lambda_{\mathrm{B}}=2 n_{0} \Lambda,
$$

where $\Lambda$ is the grating period and $n_{0}$ is the effective index of refraction.

The basic use of FBG sensors is to identify the Bragg wavelength shift. The reflected Bragg wavelength, $\lambda_{\mathrm{B}}$, is affected by the surrounding conditions. In particular, both the mechanical loading and temperature change in the FBG region induce changes in the index of refraction and grating period, resulting in a shift of the Bragg wavelength. The shift of the Bragg wavelength can be expressed in terms of the variation of the refractive index and grating period as

$$
\Delta \lambda_{\mathrm{B}}=2 \Lambda \Delta n_{0}+2 n_{0} \Delta \Lambda .
$$

The Bragg wavelength shift due to the mechanical strain $\varepsilon_{\mathrm{f}}$ is derived as follows.

The variation of the refractive index can be written as ${ }^{(15)}$

$$
\Delta n_{0}=-\frac{n_{0}^{3}}{2} \Delta\left(\frac{1}{n_{0}^{2}}\right)
$$




$$
\Delta\left(\frac{1}{n_{0}^{2}}\right)=\left[p_{12}-v_{\mathrm{f}}\left(p_{11}+p_{12}\right)\right] \varepsilon_{\mathrm{f}},
$$

where $p_{11}$ and $p_{12}$ are the Pockel's constants, and $v_{\mathrm{f}}$ is the Poisson's ratio of the optic fiber.

The strain of the FBG due to the mechanical loading is expressed as

$$
\varepsilon_{\mathrm{f}}=\frac{\Delta \Lambda}{\Lambda} .
$$

The change of the grating period can be obtained from Eq. (9).

$$
\Delta \Lambda=\varepsilon_{\mathrm{f}} \cdot \Lambda
$$

Substituting Eqs. (7) and (10) into Eq. (6) leads to

$$
\Delta \lambda_{\mathrm{B}}=2 \Lambda\left[-\frac{n_{0}^{3}}{2} \Delta\left(\frac{1}{n_{0}^{2}}\right)\right]+2 n_{0} \varepsilon_{\mathrm{f}} \cdot \Lambda .
$$

Thus, the shift of the Bragg wavelength $\Delta \lambda_{\mathrm{B}}$ due to the mechanical strain $\varepsilon_{\mathrm{f}}$ is readily determined as

$$
\Delta \lambda_{\mathrm{B}}=\left\{1-\frac{1}{2} n_{0}^{2}\left[\left(1-v_{\mathrm{f}}\right) p_{12}-v_{\mathrm{f}} p_{11}\right]\right\} \varepsilon_{\mathrm{f}} \cdot \lambda_{\mathrm{B}} .
$$

The Bragg wavelength shift due to the temperature is derived as follows. The variation of the refractive index due to the temperature change can be expressed as

$$
\Delta n_{0}=\frac{\partial n_{0}}{\partial T} \Delta T-\frac{n_{0}^{3}}{2}\left(p_{11}+2 p_{12}\right) \alpha_{\mathrm{f}} \Delta T .
$$

The variation of the grating period due to the temperature change is

$$
\Delta \Lambda=\Lambda \alpha_{\mathrm{f}} \Delta T .
$$

Substituting Eqs. (13) and (14) into Eq. (6) leads to the Bragg wavelength shift $\Delta \lambda_{\mathrm{B}}$ due to the temperature change $\Delta T$ as follows:

$$
\begin{gathered}
\Delta \lambda_{\mathrm{B}}=\left\{\left[1-\frac{1}{2} n_{0}^{2}\left(p_{11}+2 p_{12}\right)\right] \alpha_{\mathrm{f}} \Delta T+\frac{\xi}{n_{0}} \Delta T\right\} \cdot \lambda_{\mathrm{B}}, \\
\xi=\frac{\partial n_{0}}{\partial T} .
\end{gathered}
$$

Utilizing Eqs. (12) and (15), one is able to determine the mechanical strain and temperature change imposed on the optical fiber by measuring the Bragg wavelength shift. 


\section{Experimental Tests}

An FBG sensor was employed to investigate the thermal response of the optic fiber surfacebonded onto the host structure and subjected to a temperature change. The total length of the FBG sensor was $40 \mathrm{~mm}$. Only half $(20 \mathrm{~mm})$ of the FBG sensor was surface-bonded onto the test specimen; the other half of the FBG was not bonded and was free to expand. The shift of the Bragg wavelength for the nonbonded FBG induced by a temperature change can be calculated using Eq. (15). For the surface-bonded FBG sensor subjected to a temperature change of the host structure, the shift of the Bragg wavelength was modulated to take into account the thermally induced axial strain $\left(\varepsilon_{\mathrm{f}}\right)$ in the optic fiber as a result of the mismatch in the coefficients of thermal expansion between the optic fiber $\left(\alpha_{\mathrm{f}}\right)$ and the host structure $\left(\alpha_{\mathrm{h}}\right)$. The thermally induced axial strain $\left(\varepsilon_{\mathrm{f}}\right)$ was derived in $\S 2$ and presented in Eq. (4). When the FBG sensor is surface-bonded to a host structure and both experience temperature changes, the shift of the Bragg wavelength is the sum of Eqs. (12) and (15):

$$
\begin{gathered}
\Delta \lambda_{\mathrm{B}}=\left\{1-\frac{1}{2} n_{0}^{2}\left[\left(1-v_{\mathrm{f}}\right) p_{12}-v_{\mathrm{f}} p_{11}\right]\right\} \varepsilon_{\mathrm{f}} \cdot \lambda_{\mathrm{B}}+\left[1-\frac{1}{2} n_{0}^{2}\left(p_{11}+2 p_{12}\right)\right] \alpha_{\mathrm{f}} \Delta T \cdot \lambda_{\mathrm{B}}+\frac{\xi}{n_{0}} \Delta T \cdot \lambda_{\mathrm{B}}, \\
\varepsilon_{\mathrm{f}}=\frac{\left(\alpha_{\mathrm{h}}-\alpha_{\mathrm{f}}\right) \Delta T}{E_{\mathrm{f}}\left(\frac{\pi r_{\mathrm{f}}^{2}}{2 h r_{\mathrm{p}} E_{\mathrm{h}}}+\frac{1}{E_{\mathrm{f}}}\right)}\left[1-\frac{\sinh \left(\lambda_{1} L_{\mathrm{f}}\right)}{\lambda_{1} L_{\mathrm{f}} \cosh \left(\lambda_{1} L_{\mathrm{f}}\right)}\right]+\alpha_{\mathrm{f}} \Delta T .
\end{gathered}
$$

The dimensions of the test specimen, which was made of aluminum, were 210 (length) $\times 20$ (width) $\times 1 \mathrm{~mm}^{3}$ (thickness). In the experimental test, the specimen was placed in a thermal chamber and temperatures were varied from room temperature $\left(23^{\circ} \mathrm{C}\right)$ to $100{ }^{\circ} \mathrm{C}$. A thermcouple near the test specimen was used to measure the temperature change. The optic-fiber sensor system consisted of a broadband light source to illuminate the FBG sensor and an optical spectrum analyzer to detect the Bragg wavelength shift. The experimental setup is shown in Fig. 2. At room temperature, the Bragg wavelength $\left(\lambda_{\mathrm{B}}=1550.08 \mathrm{~mm}\right)$ reflected from both the bonded and nonbonded FBG sensors

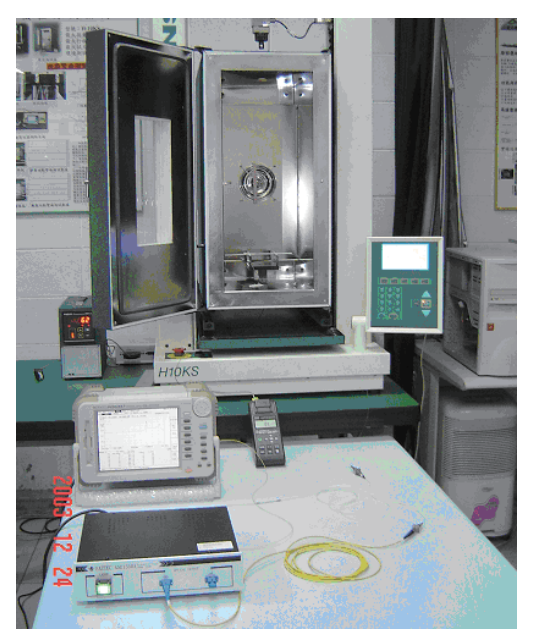

Fig. 2. (Color online) Experimental setup. 
are the same, as shown in Fig. 3(a). As the temperature was increased, the Bragg wavelength reflected from the bonded FBG became larger than that from the nonbonded FBG, as shown in Fig. 3(b). The experimental measurements of the shift of the Bragg wavelength reflected from the nonbonded and bonded FBG sensors were compared with the theoretical predictions from Eqs. (15) and (17). The Young's moduli of the optic-fiber, coating, adhesive, and the host structure were 72 $\mathrm{GPa}$, 6.7 MPa, $2 \mathrm{GPa}$, and $72 \mathrm{GPa}$, respectively. The coefficients of the thermal expansion of the optic fiber and host structure were $0.5 \times 10^{-6}$ and $23 \times 10^{-6 /}{ }^{\circ} \mathrm{C}$, respectively. The outer radii of the optic fiber and protective coating were $r_{\mathrm{f}}=62.5 \mu \mathrm{m}$ and $r_{\mathrm{p}}=125 \mu \mathrm{m}$, respectively; the thickness of the host structure $h=1 \mathrm{~mm}$. The refractive index and Pockel's constants were $n_{0}=1.45, p_{11}=0.12$, and $p_{12}=0.27$, respectively.

Comparisons of the Bragg wavelength shift predicted theoretically and experimental measurements for nonbonded and bonded FBG sensors are shown in Figs. 4 and 5, respectively. Good agreement was achieved between the theoretical predictions and experimental measurements. Tables 1 and 2 list the difference between the theoretical predictions and experimental

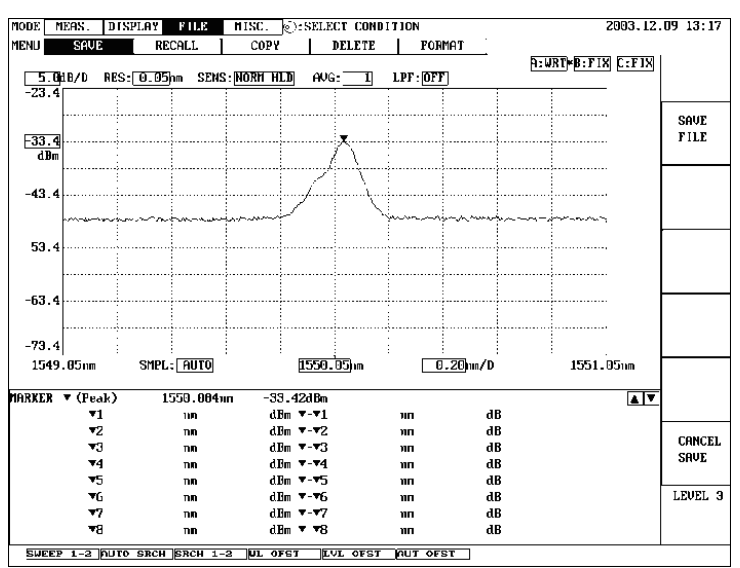

(a)

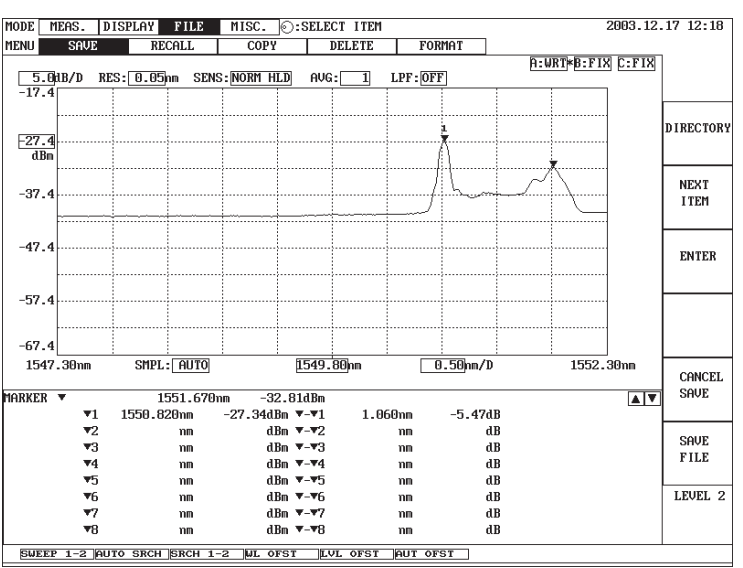

(b)

Fig. 3. Bragg wavelengths reflected from the bonded and unbonded FBG sensors. (a) At room temperature and (b) at $90{ }^{\circ} \mathrm{C}$.

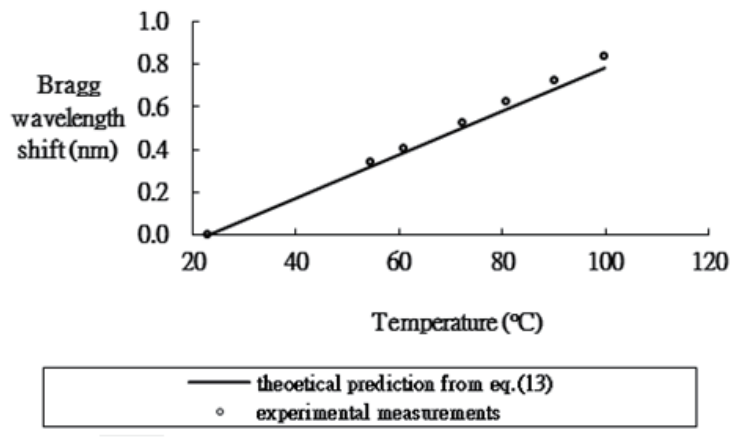

Fig. 4. Bragg wavelength shift of nonbonded FBG sensor at various temperatures.

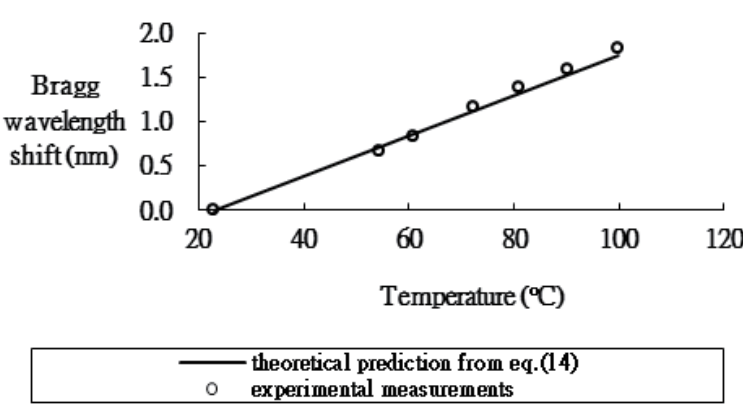

Fig. 5. Bragg wavelength shift of bonded FBG sensor at various temperatures. 
Table 1

Bragg wavelength shift of nonbonded FBG at various temperatures.

\begin{tabular}{lccc}
\hline $\begin{array}{l}\text { Temperature } \\
\left({ }^{\circ} \mathrm{C}\right)\end{array}$ & $\begin{array}{c}\text { Theoretical prediction Eq. (15) } \\
\Delta \lambda_{\mathrm{B}}(\mathrm{nm})\end{array}$ & $\begin{array}{c}\text { Experimental measurement } \\
\Delta \lambda_{\mathrm{B}}(\mathrm{nm})\end{array}$ & $\begin{array}{c}\text { Difference } \\
(\%)\end{array}$ \\
\hline 54.4 & 0.320 & 0.344 & 6.87 \\
60.9 & 0.387 & 0.408 & 5.23 \\
72.3 & 0.503 & 0.528 & 4.75 \\
80.9 & 0.591 & 0.624 & 5.33 \\
90.2 & 0.686 & 0.726 & 5.56 \\
99.8 & 0.783 & 0.836 & 6.28 \\
\hline
\end{tabular}

Table 2

Bragg wavelength shift of bonded FBG at various temperatures.

\begin{tabular}{lccc}
\hline $\begin{array}{l}\text { Temperature } \\
\left({ }^{\circ} \mathrm{C}\right)\end{array}$ & $\begin{array}{c}\text { Theoretical prediction Eq. (17) } \\
\Delta \lambda_{\mathrm{B}}(\mathrm{nm})\end{array}$ & $\begin{array}{c}\text { Experimental measurement } \\
\Delta \lambda_{\mathrm{B}}(\mathrm{nm})\end{array}$ & $\begin{array}{c}\text { Difference } \\
(\%)\end{array}$ \\
\hline 54.4 & 0.714 & 0.664 & 7.60 \\
60.9 & 0.862 & 0.840 & 2.67 \\
72.3 & 1.122 & 1.160 & 3.26 \\
80.9 & 1.318 & 1.382 & 4.62 \\
90.2 & 1.530 & 1.586 & 3.53 \\
99.8 & 1.748 & 1.836 & 4.78 \\
\hline
\end{tabular}

measurements of nonbonded and bonded FBG sensors, respectively. The maximum difference was less than $8 \%$. This result shows that the shift in the Bragg wavelength increases linearly with increasing temperature. Using the bonded and nonbonded FBG sensors, we were able to measure the thermal strain and temperature change simultaneously.

\section{Conclusions}

A close-form expression of the thermal strain in an optic fiber surface-bonded onto a host structure subjected to a temperature change is presented. The data show that the thermal strain transferred from the host material to the optic fiber depends on the bonding characteristics such as the adhesive, the coating, and the bonding length. In this study, a FBG sensor was employed to investigate the thermal response of the surface-bonded optic fiber resulting from a temperature change in the host material. Theoretical predictions of the Bragg wavelength shift were validated by the experimental measurements. The results show that theoretical predictions match the experimental test results well. The experimental test presented in this study demonstrates the capability of using bonded and nonbonded FBG sensors to evaluate thermal strain and temperature changes simultaneously.

\section{Acknowledgements}

The authors thank the Ministry of Science and Technology of the R.O.C. for financial support under grant no. NSC102-2622-E155-014-CC3. 


\section{References}

R. de Oliveira, C. A. Ramos, and A. T. Marques: Comput. Struct. 86 (2008) 340.

2 W. Ecke and K. Schroder: Proc. SPIE 6933 (2008) 693301.

3 K. T. V. Grattan and T. Sun: Sens. Actuators 82 (2000) 40.

4 L. Sorensen, T. Gmur, and J. Botsis: Composites Part A 37 (2006) 270.

5 C. Schizas and D. Karalekas: J. Mater. Process. Technol. 209 (2009) 2349.

6 S. Yashiro and T. Okabe: Composites Part A 42 (2011) 1962.

7 L. H. Kang, D. K. Kim, and J. H. Han: J. Sound Vib. 305 (2007) 534.

8 B. Torres, I. Paya-Zaforteza, P. A. Calderon, and J. M. Adam: Eng. Struct. 33 (2011) 539.

9 X. X. Li, W. X. Ren, and K. M. Bi: Sens. Actuators, A 223 (2015) 105.

10 P. Shrestha, J. H. Kim, Y. Park, and C. G. Kim: Compos. Struct. 125 (2015) 159.

11 J. Gao, J. Wu, J. Li, and X. Zhao: NDT \& E Int. 44 (2011) 202.

12 Q. Jiang, D. Hu, and M. Yang: Sens. Actuators, A 170 (2011) 62.

13 G. Zhou, L. M. Sim, and J. Loughlan: Smart Mater. Struct. 13 (2004) 1291.

14 S. C. Her and C. Y. Huang: Sensors 13 (2013) 1846.

15 J. F. Nye: Physical Properties of Crystals (Oxford University Press, Oxford, 1985). 